\title{
MicroRNA signature of end-stage idiopathic pulmonary arterial hypertension: clinical correlations and regulation of WNT signaling
}

\author{
Olivier Boucherat $^{1,2} \cdot$ Sébastien Bonnet ${ }^{1}$
}

Published online: 28 May 2016

(C) Springer-Verlag Berlin Heidelberg 2016

Pulmonary arterial hypertension (PAH) is a multifactorial and life-threatening condition characterized by abnormal remodeling of distal pulmonary arteries leading to a progressive increase in pulmonary vascular resistance and subsequent right ventricular hypertrophy and failure $[1,2]$. Despite important progress in our understanding of pathways that contribute to the excessive vasoconstriction and muscularization of pulmonary vessels, current therapies fail to substantially reduce PAH progression and mortality. Thus, PAH still remains to be a significant challenge and needs to be further investigated. Recent accumulating evidence have demonstrated that epigenetic alterations, illustrated by deregulated microRNA expression, play key roles in the onset and progression of PAH [3-5]. Although, the number of miRNAs and targets involved in PAH has grown rapidly, it is nevertheless assumed that based on the complex pathogenesis of PAH (multiple triggers affecting different cell types), the current list is not exhaustive.

In this issue, Gao and colleagues used an unbiased highthroughput approach coupled with bioinformatics tools to identify miRNAs and pathways implicated in end-stage idiopathic PAH [6]. The authors found that 21 miRNAs were differentially expressed in lung homogenates from PAH patients compared to controls. In order to interpret the biological impact of these miRNAs changes, they applied an in silico

Olivier Boucherat

olivier.boucherat@criucpq.ulaval.ca

1 Pulmonary Hypertension Research Group, Centre de Recherche de l'Institut Universitaire de Cardiologie et de Pneumologie de Québec, Université Laval, Québec, Québec, Canada

2 IUCPQ Research Centre, 2725, Chemin Sainte-Foy A2137, Québec, QC, Canada G1V 4G5 analysis of pathways based on the known miRNA target genes. Functional and pathway enrichment analysis revealed that the $\mathrm{Wmt} / \beta$-catenin pathway stands among the top ranked pathways for the target genes of all the differentially expressed miRNAs.

The Wnt signaling pathway is an evolutionary conserved system regulating multiple aspects of tissue development and homeostasis. The Wnt signaling has been broadly separated into two branches: the $\beta$-catenin-dependent (canonical) and the $\beta$-catenin-independent (non-canonical) pathways. The canonical Wnt signaling involves complex intracellular events culminating in cytoplasmic stabilization of $\beta$-catenin (through the suppression of the GSK-3 $\beta$ inhibition complex), which then translocates to the nucleus where it complexes with transcription factors and coactivators to initiate transcription of target genes [7]. During lung morphogenesis, the canonical Wnt signaling has been shown to be required for differentiation of vascular smooth muscle cells. Indeed, mice conditionally deleted for $\beta$-catenin in smooth muscle precursors displayed a thinner smooth muscle layer surrounding the developing blood vessels [8]. This anomaly was accompanied by a reduced expression of Tenascin $\mathrm{C}(\mathrm{TnC})$, an extracellular matrix molecule stimulating Pdgfr $\beta$ (a marker of smooth muscle precursors) expression. In agreement with these findings, increased expression of the $\mathrm{Wnt} / \mathrm{TnC} / \mathrm{Pdgfr}$ pathway was found in human PAH [8], indicating that this signaling axis required for pulmonary vascular smooth muscle development is aberrantly overexpressed in PAH. The implication of the Wnt signaling in PAH appears not to be restricted to smooth muscle cells. Indeed, treatment of human pulmonary artery endothelial cells (HPAECs) with WNT3 (a Wnt ligand) was shown to promote proliferation, migration, and survival of HPAECs while increasing $\beta$-catenin transcriptional activity and target genes [9], suggesting that the canonical Wnt signaling may favor the proliferation of apoptosis-resistant 
endothelial cells leading to the appearance of plexiform lesions. In agreement with these data, Gao and colleagues observed that several members of the Wnt signaling, including FZD4, FZD10, CTNNB1, and AXIN2 are upregulated in endstage idiopathic PAH lungs compared to controls. Moreover, the authors demonstrated that among the miRNAs overexpressed in PAH lungs, miR-199b-5p detected in the pulmonary vascular endothelium and adventitia positively correlates with muscularization as well as hemodynamic parameters at diagnosis such as mean pulmonary arterial pressure and pulmonary vascular resistance. In an effort to gain further insight into the role of miR-199b-5p, the authors showed that exposure of HPAECs to hypoxia (a major trigger in pulmonary hypertension) led to increased expression of miR-199b-5p and concomitant downregulation of GSK3- $\beta$, a key event in Wnt signaling activation (Fig. 1). To achieve a more direct assessment of the relationship between miR$199 \mathrm{~b}-5 \mathrm{p}$ and GSK3- $\beta$, a gain and loss of function approach was used demonstrating a significant bi-directional modulation, responding as expected to both mimic and antagomiR transfection. However, the molecular repercussions of miR199b-5p upregulation on nuclear accumulation of $\beta$-catenin and AXIN2 expression, commonly used as readouts of the Wnt pathway activity, and subsequent effects on biological processes remain to be addressed. Moreover, it would be interesting to confirm that isolated PAH-PAECs display elevated miR-199b-5p expression compared to healthy cells. Taken together, these experiments will provide valuable insights into the clinical importance of this selected miRNA. Interestingly, the findings that miR-199b overexpression sensitizes the myocardium to pathological cardiac hypertrophy whereas its silencing prevents and reverses cardiac remodeling and dysfunction indicate that elevated miR-199b-5p may accelerate heart failure in PAH [10]. In addition, miR-199b-5p was recently reported to be increased in osteosarcoma tissues and correlates with shorter overall survival, promoting proliferation of cancerous cells [11].

Given that lung biopsy in PAH patients entails substantial risk of morbidity and mortality, assessment of gene expression is based largely on lung tissues obtained from patients with end-stage disease. It is thus uncertain whether the expression changes observed in these tissues, as performed in the present study, correspond to a consequence of long-standing disease rather than mechanisms accounting for PAH development or progression. To address this problem and gain insight into the function of these miRNAs during PAH development, a longitudinal study of their expression changes in an experimental model of the disease should be conducted. Furthermore, in vivo manipulation of miRNA expression and function through the use of genetically engineered mice or local delivery of antimiRs is required to fully appreciate the potential involvement of these miRNAs in PAH pathogenesis [12-14].

It must be noted that although the expression levels of validated miRNAs in lung homogenates connects with hemodynamics and histopathological parameters, strengthening the use of miRNAs as both a therapeutic and diagnostic tool in $\mathrm{PAH}$, the pertinence of measuring miRNA levels in lung tissue for diagnostic purposes is somewhat questionable. To this end, determining whether miR-199b-5p expression is consistently increased in easy accessible body fluids from PAH patients and correlates with prognosis and severity would be of greater interest. For instance, miR-204 expression found to be decreased in human PAH and correlating with disease severity was shown to display a similar variation in buffy coat isolated from patients with PAH, supporting its use as a promising diagnostic, prognostic, and predictive biomarker $[13,15]$.

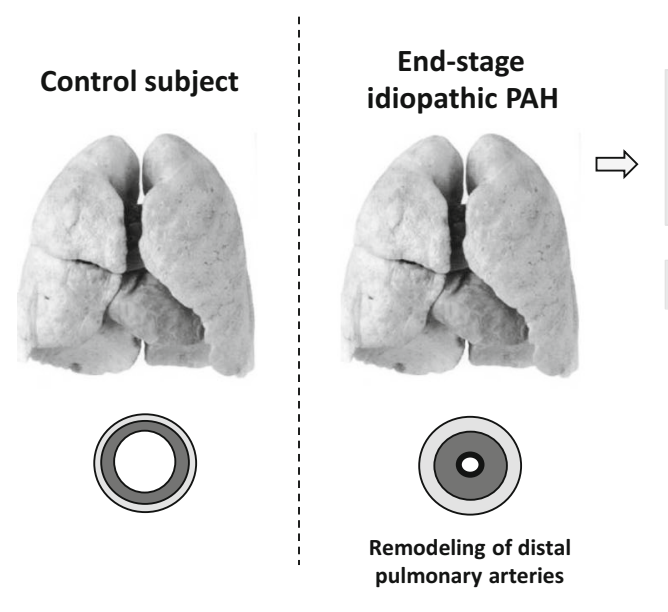

Fig. 1 Identification of a miRNA gene signature that potentially links the Wnt signaling pathway in the context of end-stage PAH. Among dysregulated miRNAs, upregulation of miR-199b-5p in pulmonary endothelial cells (PAECs) targets GSK3, inhibition of which leads to

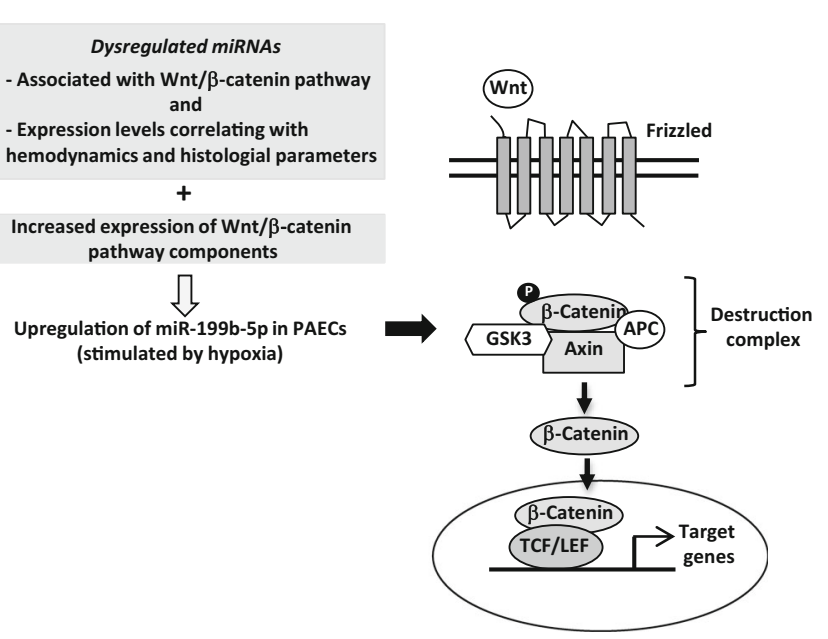

nuclear translocation of released $\beta$-catenin. Increased expression of downstream targets is proposed to favor vascular remodeling and poor outcome of PAH 
In identifying a miRNA signature that influences major PAH-related pathways, Gao and colleagues provide a starting point for a more thorough investigation. Further studies aiming to explore their underlying functions, the associated mechanisms behind their deregulation as well as their presence in extracellular fluids are warranted to establish their clinical utility.

\section{Compliance with ethical standards}

Conflict of interest The authors declare that they have conflict of interest.

\section{References}

1. Rabinovitch M (2012) Molecular pathogenesis of pulmonary arterial hypertension. J Clin Invest 122:4306-4313

2. Tuder RM, Archer SL, Dorfmüller P, Erzurum SC, Guignabert C, Michelakis E, Rabinovitch M, Schermuly R, Stenmark KR, Morrell NW (2013) Relevant issues in the pathology and pathobiology of pulmonary hypertension. J Am Coll Cardiol 62:D4-D12

3. Grant JS, White K, MacLean MR, Baker AH (2013) MicroRNAs in pulmonary arterial remodeling. Cell Mol Life Sci 70:4479-4494

4. Potus F, Graydon C, Provencher S, Bonnet S (2014) Vascular remodeling process in pulmonary arterial hypertension, with focus on miR-204 and miR-126 (2013 Grover Conference series). Pulm Circ 4(2):175-184

5. Boucherat O, Potus F, Bonnet S (2015) microRNA and pulmonary hypertension. Adv Exp Med Biol 888:237-252

6. Wu D, Talbot CC Jr, Liu Q, Jing ZC, Damico RL, Tuder R, Barnes, KC, Hassoun PM, Gao L (2016) Identifying microRNAs targeting $\mathrm{Wnt} / \beta$-catenin pathway in end-stage idiopathic pulmonary arterial hypertension. J Mol Med (Berl). doi:10.1007/s00109-016-1426-z
7. Moon RT, Kohn AD, De Ferrari GV, Kaykas A (2004) WNT and beta-catenin signalling: diseases and therapies. Nat Rev Genet 5: 691-701

8. Cohen ED, Ihida-Stansbury K, Lu MM, Panettieri RA, Jones PL, Morrisey EE (2009) Wnt signaling regulates smooth muscle precursor development in the mouse lung via a tenascin C/PDGFR pathway. J Clin Invest 119:2538-2549

9. de Jesus Perez VA, Alastalo TP, Wu JC, Axelrod JD, Cooke JP, Amieva M, Rabinovitch M (2009) Bone morphogenetic protein 2 induces pulmonary angiogenesis via Wnt-beta-catenin and WntRhoA-Rac1 pathways. J Cell Biol 184:83-99

10. da Costa Martins PA, Salic K, Gladka MM, Armand AS, Leptidis S, el Azzouzi H, Hansen A, Coenen-de Roo CJ, Bierhuizen MF, van der Nagel R et al (2010) MicroRNA-199b targets the nuclear kinase Dyrk1a in an auto-amplification loop promoting calcineurin/NFAT signalling. Nat Cell Biol 12:1220-1227

11. Zeng H, Zhang Z, Dai X, Chen Y, Ye J, Jin Z (2016) Increased expression of microRNA-199b-5p associates with poor prognosis through promoting cell proliferation, invasion and migration abilities of human osteosarcoma. Pathol Oncol Res 22: 253-260

12. Parikh VN, Jin RC, Rabello S, Gulbahce N, White K, Hale A, Cottrill KA, Shaik RS, Waxman AB, Zhang YY et al (2012) MicroRNA-21 integrates pathogenic signaling to control pulmonary hypertension: results of a network bioinformatics approach. Circulation 125:1520-1532

13. Courboulin A, Paulin R, Giguère NJ, Saksouk N, Perreault T, Meloche J, Paquet ER, Biardel S, Provencher S, Côté J et al (2011) Role for miR-204 in human pulmonary arterial hypertension. J Exp Med 208:535-548

14. Pullamsetti SS, Doebele C, Fischer A, Savai R, Kojonazarov B, Dahal BK, Ghofrani HA, Weissmann N, Grimminger F, Bonauer A et al (2012) Inhibition of microRNA-17 improves lung and heart function in experimental pulmonary hypertension. Am J Respir Crit Care Med 185:409-419

15. Meloche J, Pflieger A, Vaillancourt M, Graydon C, Provencher S, Bonnet S (2014) miRNAs in PAH: biomarker, therapeutic target or both? Drug Discov Today 19:12641269 